\title{
Uma metodologia para análise da performance de periódicos
}

\section{Guido Rummler}

\author{
Professor Titular. Universidade Estadual \\ de Feira de Santana - UEFS.
}

Este artigo apresenta uma estratégia para caracterizar periódicos de acordo com performances estabelecidas em relação aos seus congêneres. São formulados sete indicadores: índice de cobertura específica, índice de cobertura intervalar, fração de cobertura, pico de performance relativa, índice data-pró, índice de competitividade e coeficiente de equalização. Esses indicadores são definidos por relações entre dados de saída de periódicos em geral: periodicidade, número e tiragem de edições. Por meio da análise de um exemplo, são ilustradas propriedades, representações, possíveis inferências e possibilidades de aplicação desses indicadores.

Palavras-chave: Revistas; Jornais; Performance; Indicadores; Informetria; Comunicação.

\section{A metodology for analysis of periodical's performance.}

This paper presents a strategy to characterize periodicals according performances established in relation to its congeners. Seven indicators are formulated: specific covering index, intervallic covering index, covering fraction, peak of relative performance, data pro index, competitiveness index and equalizer coefficient. Those indicators are defined in relation to common periodical's output data: periodicity, number of editions and respective copies. Through a exemplified case are illustrated properties, representations, possible inferences and possibilities of use of those indicators.

Keywords: Journals; Newspapers; Performance; Indicators; Informetrics; Communication.

Recebido em 30.12.2007 Aceito em 18.06.2008 


\section{Introdução}

Independente de serem simples ou complexas as operações necessárias à representação de fenômenos por meio de indicadores, estes podem, via de regra, constituírem importantes ferramentas de avaliação a serem utilizadas em processos de tomada de decisões. De acordo com a Internacional Standardization Organization (INTERNACIONAL STANDARDIZATION ORGANIZATION - ISO, 1998 apud STRUBBS, 2004, p. 151) um indicador é

uma expressão numérica, simbólica ou verbal usada para caracterizar atividades (eventos, objetos ou pessoas) tanto em termos quantitativos como qualitativos para avaliar o valor das atividades caracterizadas e o método associado.

Essa definição reporta à possibilidade de que haja indicadores quantitativos e qualitativos, mas que, conforme analisam Baema, Cases e Medina (2005), os critérios para essa classificação possam ser conflitantes.

Para a caracterização de fenômenos relativos à informação publicada, um investigador pode contar com variado número e tipologia de indicadores, geralmente de expressão numérica, seja no âmbito da bibliometria ou da cienciometria (ou cientometria) e, mais recentemente, da webometria. Os indicadores bibliométricos, de acordo com Sen (2005), constituem uma ferramenta conceitual baseada em algum mecanismo de informação (usualmente informação bibliográfica). Muitos podem, de acordo com a expressão e descrição feita por Vinkler (1988), ser indicadores bibliométricos para propósitos cienciométricos. Isto é, serem utilizados para avaliações da atividade científica. Um outro aspecto referese ao fato de que, segundo Lopez (1996, p. 73) "Existem indicadores bibliométricos que se obtem e se interpretam facilmente. Porém, outros são bastante mais complexos de obter e manejar e devem estar reservados a especialistas".

Por outro lado, a informetria constitui um campo mais abrangente que a bibliometria e a cienciometria, na medida em que, segundo MaciasChapula (1998, p. 135), estuda "aspectos quantitativos da informação em qualquer formato, e não apenas registros catalográficos ou bibliografias, referentes a qualquer grupo social, e não apenas aos cientistas". Igual abrangência encontra-se no dizer de Tague-Sutcliffe, citado por Vanti (2005, p. 155), atribuindo à informetria o "analisar também os processos de comunicação informal, inclusive falada, e dedicar-se a pesquisar os usos e necessidades de informação de grupos sociais desfavorecidos, e não só das elites intelectuais". É nessa amplitude de fronteiras que se situa o presente trabalho, na medida em que as ferramentas aqui elaboradas aplicam-se à análise de questões relativas à veiculação impressa de conteúdos de qualquer natureza, baseando-se em parâmetros comuns que caracterizam a emissão periódica dos respectivos suportes. Desse modo, os indicadores propostos tanto poderão servir ao 
pesquisador científico quanto para subsidiar decisões de cunho econômico-mercadológico.

\section{Formulação dos indicadores}

É senso comum considerar que, quanto mais exemplares de um jornal ou revista sejam postos em circulação, maior poderá ser a disseminação de seus conteúdos. Em contraposição, há o fato de que, via de regra, cada periódico divide ou compete com outros por penetrabilidade na população-alvo. Assim, seu alcance é relativo à abrangência dos demais, podendo isto estar refletido nas respectivas diferenças de tiragens e/ou número de edições. Estes parâmetros, comuns a todos os periódicos, podem ser utilizados para caracterizá-los conforme se segue.

\subsection{Medida relativa a uma edição}

Estimar o alcance de um periódico, pela proporção do número de seus exemplares em relação ao número total de exemplares de todos os periódicos de cobertura referentes a uma área geográfica ou de conhecimento, constitui um erro cuja margem pode ser aproximadamente igual a de seus congêneres assim tratados e, portanto, uma razoável estimativa, expressa em termos relativos, de sua cobertura.

Como a quantidade de exemplares é representada por sua tiragem, a proporção desta, referida à de todos os periódicos de cobertura, corresponde ao $\frac{t_{x}}{t_{c}}$ quociente, onde $t_{x}$ é a tiragem de uma edição do periódico ${ }^{t_{c}}$ considerado e $t_{c}$ a soma das tiragens de todos os periódicos de cobertura, incluindo $t_{x}$, ocorrentes no período de vigência daquela edição. Essa proporcionalidade expressa em termos percentuais constitui o que será denominado de índice de cobertura específica - ICE, sendo assim representado:

$$
\mathrm{ICE}=\frac{100 \mathrm{t}_{\mathrm{x}}}{\mathrm{t}_{\mathrm{C}}}
$$

\subsection{Medida relativa ao ciclo editorial}

Quando uma medida referir-se a qualquer número de edições de um periódico e for considerada sua proporcionalidade em relação à soma das tiragens de todos os periódicos de cobertura ocorrentes no ciclo editorial do periódico $x$, ter-se-á o que será denominado de fração de cobertura FC, cuja expressão é:

$$
\mathrm{FC}=\frac{100 \mathrm{~T}_{\mathrm{X}}}{\mathrm{T}_{\mathrm{CC}}}
$$

Onde $T_{x}$ é a tiragem da(s) edição(ões) referida(s) de $x$, e $T_{c c}$ a soma das tiragens de todas as edições de todos os periódicos de cobertura ocorrentes no ciclo editorial de $\mathrm{x}$. 


\subsection{Medida relativa a um intervalo}

Para uma análise que reporte a determinado intervalo de tempo, deve ser considerada a possibilidade de ocorrerem diferenças quanto ao número de edições e tiragens praticadas em diversos intervalos. Assim, não lhe pode corresponder o índice de cobertura específica.

Tendo-se:

$\mathrm{T}_{\mathrm{xi}}=$ soma das tiragens do periódico $\mathrm{x}$ correspondentes às edições ocorrentes no intervalo em questão;

$\mathrm{T}_{\mathrm{ci}}=$ soma das tiragens das edições de todos os periódicos de cobertura, ocorrentes no mesmo intervalo. À relação abaixo estabelecida denominar-se-á índice de cobertura intervalar ICI do periódico $x$, tendo por expressão:

$$
\mathrm{ICI}=\frac{100 \mathrm{~T}_{\mathrm{xi}}}{\mathrm{T}_{\mathrm{ci}}}
$$

Caso se queira estabelecer o índice de cobertura intervalar para um grupo de periódicos, a soma da tiragem destes será representada por $\Sigma T x i$, conferindo ao ICI a seguinte expressão genérica:

$$
\mathrm{ICI}=\frac{100 \sum \mathrm{T}_{\mathrm{xi}}}{\mathrm{T}_{\mathrm{ci}}}
$$

Para identificar a situação particular em que o intervalo de referência respectivo a um ICI corresponde a um ciclo completo de periodicidade de $x$, será atribuído a esse indicador a denominação de índice de cobertura cíclica - ICC daquele periódico.

Pode-se também ter ICC de um grupo de periódicos $x$, desde que todos desse grupo tenham ciclo editorial referido à mesma unidade de divisão cronológica. No caso do exemplo adiante apresentado, TAB.1 e 2, os três periódicos têm cronologia igualmente referida a dias da semana.

\subsection{Realce de performance}

Quando não forem iguais as tiragens das edições de algum periódico de cobertura, poderão ser encontradas diversas medidas ao longo dos respectivos ciclos editoriais referentes aos indicadores mencionados. Desse modo, ocorrerão situações que representem favorecimento relativo a determinadas edições de cada um.

\subsubsection{Pico de performance relativa}

Para efeito de identificação, sobre o que refere esse item, para o valor de ICE e/ou ICI respectivo à condição mais favorável, representada por ICE, ICI, IC e CE, será usada a designação pico de performance relativa - PPR. Portanto, PPR $=\mathrm{ICE}_{\text {máx }}$ e/ou $\mathrm{ICI}_{\text {máx }}$ 


\subsection{2 Índice data-pró}

Para fins de situar cronologicamente a ocorrência do PPR, para o intervalo do ciclo editorial em que este ocorre, será usada a designação de índice data-pró - IDP. Assim, o IDP é representado por dia-de-semana, semana do mês, quinzena, mês, semestre, etc.

\subsection{Competitividade}

Indicadores que definam o posicionamento de um ou mais periódicos frente à condição dos demais concorrentes podem ser matematicamente expressos pelo mesmo recurso de medida da proporcionalidade entre tiragens das respectivas edições. Duas dessas representações são formuladas conforme segue:

\subsection{1 Índice de competitividade - IC}

A medida desse índice é expressa, percentualmente, por:

$$
\mathrm{IC}=\frac{100\left(\sum \mathrm{T}_{\mathrm{x}}-\sum \mathrm{T}_{\mathrm{c}-\mathrm{x}}\right)}{\sum \mathrm{T}_{\mathrm{c}-\mathrm{x}}}
$$

onde $T_{x}$ representa a tiragem total de uma ou mais edições de um periódico $x$, e $T_{c-x}$ a tiragem de todos os demais periódicos no período de vigência da(s) edição(ões) de $x$.

\subsubsection{Coeficiente de equalização - CE}

É uma medida que expressa em termos percentuais, positivos ou negativos, a proporção de aumento ou diminuição que o(s) periódico(s) $x$ deve $(m)$ sofrer, nas tiragens do intervalo considerado, para igualar(em)se aos concorrentes quanto à medida dos indicadores precedentes. Sua expressão matemática, de acordo com os parâmetros identificados no item anterior, é:

$$
\mathrm{CE}=\frac{100\left(\Sigma \mathrm{T}_{c-x}-\Sigma \mathrm{T}_{\mathrm{x}}\right)}{\Sigma \mathrm{T}_{\mathrm{x}}}
$$

\section{Resultados}

No quadro sinóptico adiante formulado, são discriminados os indicadores, sua expressão e possíveis inferências quanto ao que possam representar no contexto informacional de uma área. A TAB. 1 apresenta dados de um exemplo hipotético, baseado em situação existente em uma unidade federativa brasileira, visando exemplificar a aplicação dos diversos indicadores antes definidos. Os resultados obtidos encontram-se na TAB. 2. Nessa tabela, os diversos valores resultantes da aplicação das fórmulas para os cálculos de ICE, FC, ICI, IC e CE, como as indicações do PRP e IDP, ilustram: 
Nos casos 1, 2 e 3, as medidas de ICE representam os percentuais correspondentes a cada periódico frente ao número de exemplares emitidos simultaneamente por um ou mais periódicos de cobertura da área. São medidas referentes a cada edição apontada dos periódicos $A, B$ e C, estabelecendo uma faixa de variação de zero a 60,0\%;

Nos casos 1 a 12, as medidas do FC referentes a uma ou mais edições de um periódico $x$ representam estimativas da proporção de cobertura que este detém relativamente à tiragem de todos os periódicos, ocorrida durante o ciclo de periodicidade de " $x$ ". Para essas medidas a faixa de variação encontrada foi de zero a $45,2 \%$;

nos casos 4 a 12, medidas do ICI correspondentes aos períodos assinalados, variando de $10,1 \%$ a $50,7 \%$, representam estimativas dos percentuais de cobertura dada pelas respectivas edições, relativamente a todas que ocorreram nos correspondentes períodos. Nos casos 11 e 12 medem-se também índices intervalares para periódicos agrupados.

Nos casos 1 e 10, medidas do PRP referentes aos periódicos A, B e C, correspondem a $60 \%, 40 \%$ e $21,4 \%$, respectivamente. Essas medidas, ocorrendo nos domingos para os periódicos A e B e qualquer dia, de segunda à sexta-feira, para o periódico $C$, apontam tais momentos como sendo os que correspondem aos respectivos índices data-pró (IDP). Medidas que variam de positivas a negativas $(+50,0 \%$ a $-88,7 \%)$, correspondentes ao IC de cada periódico, indicam em que proporção da tiragem dos demais periódicos cada um deles encontra-se em vantagem ou desvantagem frente àqueles. Uma também possível variação, positiva ou negativa $(-33,3 \%$ a $+526,7 \%)$, refere-se ao coeficiente de equalização - CE. Este indica, por exemplo, que, se o periódico A diminuísse em $33,3 \%$ a sua tiragem de domingo, igualaria a presencialidade dessa sua edição com a dos que concorrem nesta data. Situação de extremo oposto corresponde ao periódico C (caso 5), que deve aumentar em 526,7\% o número dos exemplares que edita nas sextas e sábados para, nesse período, ter um grau de exposição igual ao dos demais.

\section{QUADRO SINÓPTICO}

Indicadores formulados e suas representações

\begin{tabular}{|c|c|c|}
\hline \multicolumn{3}{|c|}{1 ÍNDICE DE COBERTURA ESPECÍFICA } \\
\hline \multirow{3}{*}{$\mathrm{ICE}=$} & $100 t_{x}$ & 100 (tiragem de uma edição "x" do periódico) \\
\hline & $=\mathrm{t}_{\mathrm{C}}$ & tiragem de todos periódicos de cobertura no período de vigência de "x" \\
\hline & & $\begin{array}{l}\text { Inferência: estimativa de proporção da fatia do mercado consumidor } \\
\text { para a edição "x" do periódico. }\end{array}$ \\
\hline \multicolumn{3}{|c|}{2 FRAÇÂO DE COBERTURA } \\
\hline \multirow{3}{*}{$\mathrm{FC}=$} & $100 \mathrm{~T}_{\mathrm{xc}}$ & 100 (total de tiragem(ns) de uma ou mais edições do periódico " $\mathrm{x}$ ") \\
\hline & $\mathrm{T}_{\mathrm{cc}}$ & soma das tiragens dos periódicos de cobertura no ciclo de " $\mathrm{x}$ " \\
\hline & & $\begin{array}{l}\text { Inferência: estimativa do grau de visibilidade que detém um periódico } \\
\text { referente a seu ciclo editorial completo, relativamente ao conjunto dos } \\
\text { periódicos desta área. }\end{array}$ \\
\hline
\end{tabular}




\section{QUADRO SINÓPTICO}

\section{Indicadores formulados e suas representações}

(continuação)

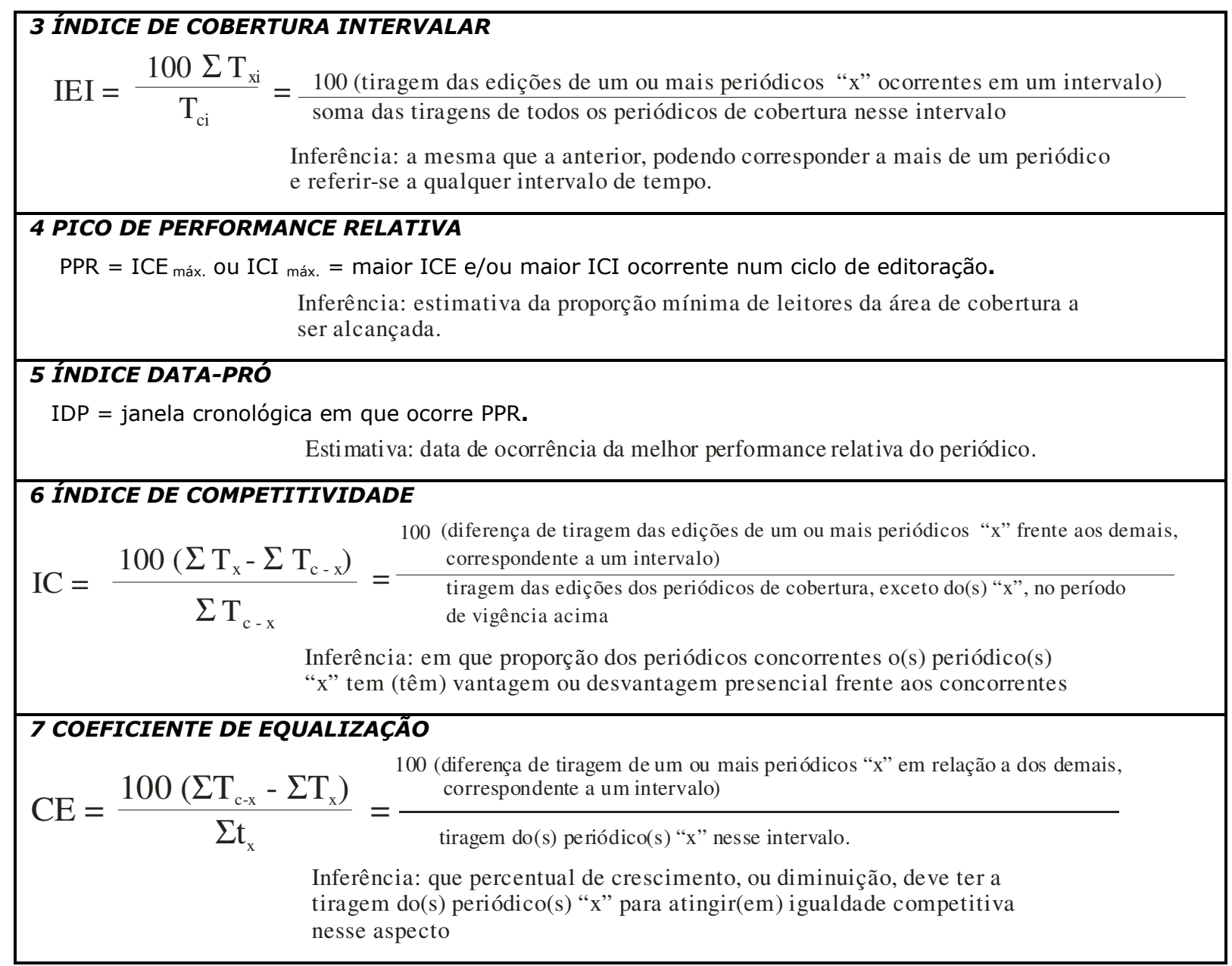

TABELA 1: Tiragens por edição dos periódicos de uma área, referentes ao exemplo para análise

\begin{tabular}{|c|c|c|c|}
\hline \multirow{3}{*}{ Periódico } & \multicolumn{3}{|c|}{ Edição } \\
\hline & $2^{a}$ a $6^{a}$ feira & Sábado & Domingo \\
\hline & $\begin{array}{l}\text { tiragem/edição } \\
\text { (x 1000) }\end{array}$ & $\begin{array}{l}\text { tiragem/edição } \\
\text { (x 1000) }\end{array}$ & $\begin{array}{c}\text { tiragem/edição } \\
\text { (x 1000) }\end{array}$ \\
\hline $\bar{A}$ & 30 & 30 & 45 \\
\hline B & 25 & 28 & 30 \\
\hline $\mathbf{C}$ & 15 & 15 & 0 \\
\hline
\end{tabular}

Fonte: Dados da pesquisa. 


\section{TABELA 2}

Medidas do índice de cobertura específica (ICE), fração de cobertura (FC), índice de cobertura intervalar (ICI), pico de performance relativa (PPR), índice de competitividade (IC) e coeficiente de equalização (CE) de cada periódico, ou agrupamento de periódicos, caracterizados na Tabela 1, referentes a diversas alternativas de um ciclo de periodicidade.

\begin{tabular}{|c|c|c|c|c|c|c|c|c|c|c|c|c|c|c|c|}
\hline \multirow{3}{*}{$\begin{array}{l}\text { : } \\
\text { ֻू }\end{array}$} & \multirow{3}{*}{$\begin{array}{l}\text { : } \\
\frac{0}{2} \\
\frac{0}{0} \\
0\end{array}$} & \multicolumn{7}{|c|}{ Edição } & \multicolumn{7}{|c|}{ Indicadores } \\
\hline & & \multirow{2}{*}{ 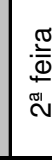 } & \multirow{2}{*}{ 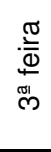 } & \multirow{2}{*}{ 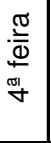 } & \multirow{2}{*}{ 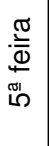 } & \multirow{2}{*}{ 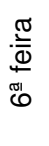 } & \multirow{2}{*}{ 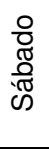 } & \multirow{2}{*}{ 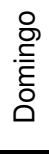 } & \multirow{2}{*}{ ICE (\%) } & \multirow{2}{*}{ FC (\%) } & \multicolumn{2}{|c|}{$\mathrm{ICl}(\%)$} & \multirow[t]{2}{*}{ PPR (\%) } & \multirow[t]{2}{*}{ IC (\%) } & \multirow[t]{2}{*}{ CE (\%) } \\
\hline & & & & & & & & & & & Unitário & Conjunto & & & \\
\hline \multirow{3}{*}{1} & $\mathrm{~A}^{*}$ & & & & & & & $\mathrm{x}$ & $60,0^{\star}$ & 9,0 & & & 60 & $50,0^{*}$ & $-33,3^{*}$ \\
\hline & $\mathrm{B}^{*}$ & & & & & & & $x$ & $40,0^{*}$ & 6,0 & & & 40 & $-33,3^{\star}$ & $50,0^{*}$ \\
\hline & C & & & & & & & & 0 & 0 & & & & 1 & 1 \\
\hline \multirow{3}{*}{2} & $\mathrm{~A}$ & & & & & & $x$ & & 41,1 & 6,0 & & & & $-30,2$ & 43,3 \\
\hline & $B$ & & & & & & $x$ & & 38,4 & 5,6 & & & & $-37,8$ & 96,4 \\
\hline & $\mathrm{C}$ & & & & & & $x$ & & 20,5 & 3,0 & & & & $-74,1$ & 286,7 \\
\hline \multirow{3}{*}{3} & A & & & & & $x$ & & & 42,9 & 6,0 & & & & $-25,0$ & 33,3 \\
\hline & $\mathrm{B}$ & & & & & $x$ & & & 35,7 & 5,0 & & & & $-44,4$ & 80,0 \\
\hline & $\mathrm{C}^{*}$ & & & & & $x$ & & & $21,4^{*}$ & 3,0 & & & 21,4 & $-72,7$ & 266,7 \\
\hline \multirow{3}{*}{4} & $\mathrm{~A}$ & & & & & & $x$ & $x$ & & 15,1 & 50,7 & & & 2,7 & $-2,7$ \\
\hline & $B$ & & & & & & $x$ & $\mathrm{x}$ & & 11,6 & 39,2 & 100 & & $-35,6$ & 55,2 \\
\hline & $\mathrm{C}$ & & & & & & $x$ & & & 3,0 & 10,1 & & & $-88,7$ & 486,7 \\
\hline \multirow{3}{*}{5} & $A$ & & & & & $\mathrm{x}$ & $x$ & $\mathrm{x}$ & & 21,1 & 48,2 & & & $-7,1$ & 7,6 \\
\hline & $B$ & & & & & $x$ & $x$ & $x$ & & 16,7 & 38,1 & 100 & & $-38,5$ & 62,7 \\
\hline & $\mathrm{C}$ & & & & & $x$ & $x$ & & & 6,0 & 13,8 & & & $-84,0$ & 526,7 \\
\hline & $\bar{A}$ & & & & $\mathrm{x}$ & $\bar{x}$ & $\bar{x}$ & $\mathrm{x}$ & & 27,1 & 46,9 & & & $-11,8$ & 13,3 \\
\hline 6 & B & & & & $x$ & $x$ & $x$ & $x$ & & 21,7 & 37,5 & 100 & & $-40,0$ & 66,7 \\
\hline & $\mathrm{C}$ & & & & $x$ & $x$ & $x$ & & & 9,0 & 15,6 & & & $-81,5$ & 440,0 \\
\hline & $\bar{A}$ & & & $x$ & $x$ & $x$ & $x$ & $x$ & & 33,1 & 46,1 & & & $-24,1$ & 19,4 \\
\hline 7 & B & & & $x$ & $x$ & $x$ & $x$ & $x$ & & 26,7 & 37,2 & 100 & & $-40,9$ & 69,2 \\
\hline & $\mathrm{C}$ & & & $x$ & $x$ & $x$ & $x$ & & & 12,0 & 16,8 & & & $-79,9$ & 396,7 \\
\hline & $\mathrm{A}$ & & $x$ & $x$ & $x$ & $x$ & $x$ & $x$ & & 39,2 & 45,6 & & & $-16,3$ & 19,5 \\
\hline 8 & $\mathrm{~B}$ & & $\bar{x}$ & $\bar{x}$ & $\bar{x}$ & $\bar{x}$ & $\bar{x}$ & $\bar{x}$ & & 31,7 & 36,9 & 100 & & $-41,5$ & 70,9 \\
\hline & $\mathrm{C}$ & & $x$ & $x$ & $x$ & $x$ & $x$ & & & 15,1 & 17,5 & & & $-78,8$ & 370,7 \\
\hline & $\mathrm{A}$ & $x$ & $x$ & $x$ & $x$ & $x$ & $x$ & $x$ & & 45,2 & 45,2 & & & $-17,6$ & 21,3 \\
\hline $9^{* *}$ & $B$ & $x$ & $x$ & $x$ & $x$ & $x$ & $x$ & $x$ & & 36,7 & 36,7 & 100 & & $-41,9$ & 72,1 \\
\hline & $\bar{C}$ & $x$ & $x$ & $x$ & $x$ & $x$ & $x$ & & & 18,1 & 18,1 & & & $-77,9$ & 353,3 \\
\hline & $A$ & $x$ & $x$ & $x$ & $x$ & $x$ & & & & 30,1 & 42,9 & & & $-25,0$ & 33,3 \\
\hline 10 & $B$ & $x$ & $x$ & $x$ & $x$ & $x$ & & & & 25,1 & 35,7 & 100 & & $-44,4$ & 80,0 \\
\hline & $\mathrm{C}^{*}$ & $x$ & $x$ & $x$ & $x$ & $x$ & & & & 15,1 & $21,4^{\star}$ & & 21,4 & $-72,7^{*}$ & $266,7^{*}$ \\
\hline & $\mathrm{A}$ & $x$ & & & $x$ & & & $x$ & & 21,1 & 48,8 & 86.0 & & $-4,5$ & 4,8 \\
\hline 11 & B & $x$ & & & $x$ & & & $x$ & & 16,1 & 37,2 & 86,0 & & $-40,7$ & 68,8 \\
\hline & $\mathrm{C}$ & $\bar{x}$ & & & $\bar{x}$ & & & & & 6,0 & 14,0 & 1 & & $-83,8$ & 516,7 \\
\hline & $A$ & $x$ & & & $x$ & & & $\mathrm{x}$ & & 21,1 & 48,8 & 1 & & $-4,5$ & 4,8 \\
\hline 12 & $B$ & $x$ & & & $x$ & & & $x$ & & 16,1 & 37,2 & & & & \\
\hline & $\mathrm{C}$ & $x$ & & & $x$ & & & & & 6,0 & 14,0 & 51,2 & & 4,8 & $-4,5$ \\
\hline
\end{tabular}

$X$ - edição considerada.

* situação de melhor performance relativa do periódico, que refere o pico de performance relativa - PPR, correspondendo ao maior valor dentre os $I C E$ e $I C I$.

${ }^{* *}$ situação correspondente ao índice de cobertura cíclica - ICC.

\section{Fonte: Dados da pesquisa.}




\section{Considerações}

Frente ao objetivo de disseminar um conteúdo x (um artigo, um edital, um anúncio etc.) por meio de sua publicação em periódicos, há um elenco de variáveis intervenientes, que se referem desde a aspectos de trânsito dos suportes de veiculação no âmbito da população-alvo até a questões econômico-mercadológicas. Assim, diversos fatores conspiram para, ou favorecem a desejada visibilidade de $\mathrm{x}$.

A busca de indicadores cujas medidas possam ser estabelecidas a partir de parâmetros menos difíceis de obter, tais como o número de edições e as respectivas tiragens, pode garantir a caracterização de performances comparativas de razoável precisão, permitindo, por outro lado, fazer ilações referentes a aspectos tais como a cobertura, a exposição, a potencialidade informativa e a competitividade. Isso se deve ao fato dos indicadores propostos caracterizarem relações (proporcionalidade), e não medidas absolutas desses fenômenos.

Portanto, as diferenças estabelecidas em função da periodicidade, número e tiragem de edições, constituem vetores de desproporcionalidades características de uma condição basal, ou performance de saída. As conseqüentes medidas expressas a partir dessas condições podem representar estimativas quanto ao possível grau de informação a ser alcançado, visto que sua efetiva constatação empírica é de difícil execução.

Feitas tais ressalvas, pode-se trazer como exemplo pragmático de um possível emprego desses indicadores a seguinte situação: saber se a diferença percentual no valor cobrado pela inserção de uma matéria em uma revista (ou em várias revistas) comparativamente ao de outra(s) corresponde à estimativa de exposição que esta terá no contexto de uma área. Ou então, em que momento o periódico tem maior probabilidade de estar proporcionalmente mais exposto. Ou ainda, que ajuste poderia favorecer uma equalização na questão custo/benefício?

Uma aproximação a essas respostas poderia vir da aplicação, dependente das circunstâncias desejadas, dos índices de cobertura para a primeira pergunta, do pico de performance relativa para a segunda pergunta e do índice de equalização para a terceira questão.

Por outro lado, aspectos ou propriedades dos indicadores formulados podem ser observados na TAB. 2:

- a fração de cobertura - FC correspondente a diversas edições equivale ao somatório das edições individuais (casos 4 a 12). Equivale também ao ICI e ICC quando for referido a todas as edições de um ciclo de periodicidade (caso 9).

- o índice de cobertura intervalar - ICI equivale ao índice de cobertura específica - ICE, quando este apresentar a mesma medida para cada edição que ocorra nesse intervalo (caso 3 x caso 10); 
- o índice de cobertura intervalar - ICI de um agrupamento de periódicos equivale à soma desses índices referentes a cada periódico do grupo (casos 11 e 12)

- o índice de competitividade - IC e o coeficiente de equalização - CE não são complementares, embora se refiram a uma mesma condição; isto porque o primeiro é dado em relação à pujança dos competidores e o segundo em relação a si próprio.

\section{Considerações finais}

As diversas medidas utilizadas na caracterização de performances de periódicos são aqui propostas como indicadores, visto que, conforme define van Raan (2004), resultam de uma operação matemática (geralmente aritmética simples) com dados referentes aos mesmos, que remete significados. Na medida em que relacionam parâmetros comuns a periódicos impressos de qualquer natureza, sua classificação como indicadores informétricos é compatível com a abrangência desta área, conforme referido na introdução desse estudo. Pode-se ainda caracterizálos como estocásticos quando relacionados a probabilidades de efeitos gerados a partir da condição basal que caracterizam. Isto é, caso se aceite, por exemplo, o percentual medido pelo ICE ou ICI como equivalente ao potencial informativo do periódico no respectivo intervalo.

Tomando como exemplo de como são classificados por Vinkler (2001) os indicadores cienciométricos, cabe a seis dos aqui apresentados a categoria de indicadores relativos, visto que suas medidas são relativizadas a um conjunto específico de periódicos congêneres. Ao índice data-pró - IDP caberia, de acordo com uma classificação apresentada por Sen (2005), a categorização de indicador derivado, visto apoiar-se na medida do pico de performance relativa - PPR.

Para os indicadores formulados, pode-se também reconhecer os cinco pontos fundamentais estabelecidos nas normas ISO 11620, de 1998 (apud STRUBBS, 2004), referentes a indicadores de desempenho, visto que: provêem informação para tomar decisões, estabelecer metas, priorizar atividades e serviços; produzem o mesmo resultado sob as mesmas circunstâncias; medem o que se pretende medir; são apropriados à proposta que se quer aplicar, isto é, as unidades, as escalas e as operações necessárias ao processo de medição são compatíveis com o procedimento requerido do aplicador. E, por fim, são práticos, no sentido de que usam dados disponíveis, são razoáveis quanto ao esforço, tempo, custo e paciência necessária para sua operacionalização.

\section{Referências}

BAEMA, A. J. L.; CASES, M. V.; MEDINA, M. B. Indicadores cuantitativos y cualitativos para la evaluación de la actividad investigadora: ¿complemetarios? ¿contradictorios? ¿excluyentes?. Cuadernos IRC. 
Disponível em: <http://www.uca.es/serv/consejo social/doc/ transferencia14.pdf >. Acesso em: 28 jun. 2005.

INTERNACIONAL STANDARDIZATION ORGANIZATION. Information and documentation library performance indicators. Geneve, [s.n.], 1998. 56p. citado por STRUBBS, E. A. Indicadores de desenpeño: naturaleza, utilidad y construcción. Ciência da Informação, v. 33, n. 1, p. 149-154, 2004.

LÓPEZ, P. L. Introducción a la bibliometría. Valencia (ES):Promolibro, 1996. 128p.

MACIAS-CHAPULA, C. A. O papel da informetria e da cienciometria e sua perspectiva nacional e internacional. Ciência da Informação, v. 27, n. 2, p. 134-140, 1998.

SEN, S. K. For what purpose are the bibliometric indicators and how should they work. Disponivel em: $<$ http://www.ricyt.org/interior/normalizacion/IV taller/sen.pdf $>$ Acesso em: 18 jul. 2005.

STRUBBS, E. A. Indicadores de desenpeño: naturaleza, utilidad y construcción. Ciência da Informação, v. 33, n. 1, p. 149-154, 2004.

TAGUE-SUTCLIFFE, J. An introduction to informetrics. Information Processing \& Management, v. 28, n. 1, p. 1-3, 1992 citado por VANTI, N. A. P. Da bibliometria à webometria: uma exploração conceitual dos mecanismos utilizados para medir o registro da informação e a difusão do conhecimento. Ciência da Informação, v. 31, n. 2, p. 152-162, 2002.

van RAAN, A. F. J. Measuring science. In: MOED, H. F.; GLÄNZEL, W.; $\mathrm{SCHMOCH}, \mathrm{U}$. (edits.). Handbook of quantitative science and technology research. Dordrecht: Kluver Academic Publishers, 2004. p.19-50.

VANTI, N. A. P. Da bibliometria à webometria: uma exploração conceitual dos mecanismos utilizados para medir o registro da informação e a difusão do conhecimento. Ciência da Informação, v. 31, n. 2, p. 152-162, 2002.

VINKLER, P. An attenmpt of surveying and classifying bibliometric indicators for scientometric purposes. Scientometrics, v. 13, n. 5-6, p. 239-259, 1988.

VINKLER, P. An attempt for defining some basic categories of scientometrics and classifying the indicators of evaluative scientometrics. Scientometrics, v. 50, n. 3, p. 539-544, 2001. 